T. Kanzaki

Nagoya Math. J.

Vol. 49 (1973), 127-141

\title{
ON THE QUADRATIC EXTENSIONS AND THE EXTENDED WITT RING OF A COMMUTATIVE RING
}

\author{
TERUO KANZAKI
}

Let $B$ be a ring and $A$ a subring of $B$ with the common identity element 1. If the residue $A$-module $B / A$ is inversible as an $A$-Abimodule, i.e. $B / A \otimes_{A} \operatorname{Hom}_{A}(B / A, A) \approx \operatorname{Hom}_{A}(B / A, A) \otimes_{A} B / A \approx A$, then $B$ is called a quadratic extension of $A$. In the case where $B$ and $A$ are division rings, this definition coincides with in P. M. Cohn [2]. We can see easily that if $B$ is a Galois extension of $A$ with the Galois group $G$ of order 2, in the sense of [3], and if $\operatorname{Tr}_{G}(B)=\left\{\sum_{\sigma \in G} \sigma(b): b \in B\right\}=A, B$ is a quadratic extension of $A$. $A$ generalized crossed product $\Delta(f, A, \Phi$, $G$ ) of a ring $A$ and a group $G$ of order 2, in [4], is also a quadratic extension of $A$.

In this note, we study the case of commutative quadratic extensions, where $A$ is a commutative ring and $B$ is an $A$-algebra. Let $A$ be a commutative ring with the identity element 1 . We shall say that $B$ is a quadratic extension of $A$ if $B$ is a ring extension of $A$ with the common identity element and $B$ is a finitely generated projective $A$-module of rank 2 so that $B$ is a commutative ring. We denote by $Q(A)$ (resp. $Q_{s}(A)$ ) the set of all $A$-algebra isomorphism classes of quadratic (resp. separable quadratic) extensions of $A$. It is known that $Q_{s}(A)$ forms a group under a certain product, and in [1], [6] and [7], the group $Q_{s}(A)$ is investigated. In this note, in $\S 1$, we define a product in $Q(A)$, which coincides with the product defined in [1], [6] and [7] in the subset $Q_{s}(A)$. Then, $Q(A)$ forms an abelian semi-group containing the subsemi-group $Q_{s}(A)$ which is a group, and an element $[B]$ in $Q(A)$ is contained in $Q_{s}(A)$ if and only if $[B]^{2}=[B][B]$ is the identity element of $Q(A)$. In $\S 2$, we give a generalization of a quadratic module and define $A$-isomorphisms between them. Then, we can consider a category consisting of these

Received April 24, 1972.

Revised September 4, 1972. 
extended quadratic modules and $A$-isomorphisms. From this category we can construct a commutative ring $W^{*}(A)$. In $\S 3$, we shall show that $W^{*}(A)$ is a commutative ring with the identity element, and there exists a ring homomorphism of the Witt ring $W(A)$ to $W^{*}(A)$ for which the image is an ideal of $W^{*}(A)$. Especially, if 2 is inversible in $A$, then $W(A)$ and $W^{*}(A)$ are isomorphic. In $\S 4$, we shall give a group homomorphism of $Q_{s}(A)$ to the unit group $U\left(W^{*}(A)\right)$ of $W^{*}(A)$.

\section{Quadratic extension.}

Let $A$ be an arbitrary commutative ring with the identity element 1 . A commutative extension ring $B$ of $A$ is called a quadratic extension of $A$ if $B$ is a finitely generated projective $A$-module of rank 2 and $B$ has the same identity element 1 . If $B$ is a quadratic extension of $A$, then there exist a finitely generated projective $A$-module $U$ of rank 1 and quadratic forms $q: U \rightarrow A$ and $q^{\prime}: U \rightarrow U^{1)}$ such that $B=A \oplus U$ and $x^{2}=q(x)+q^{\prime}(x)$ for all $x$ in $U$.

LEMMa 1. Let $U$ be a finitely generated projective A-module of rank 1 , and $q^{\prime}: U \rightarrow U$ a quadratic form. Then there exists an A-homomorphism $f: U \rightarrow A$ such that $q^{\prime}(x)=f(x) x$ for all $x$ in $U$.

Proof. For the quadratic form $q^{\prime}: U \rightarrow U$, there exists a bilinear form $B: U \times U \rightarrow U$ such that $q^{\prime}(x)=B(x, x)$ for all $x$ in $U$, (cf. (2.3) in [2]). We may consider that $B$ is an element in $\operatorname{Hom}_{A}\left(U \otimes_{A} U, U\right)$. Then by the following natural isomorphisms; $\operatorname{Hom}_{A}\left(U \otimes_{A} U, U\right) \approx$ $\operatorname{Hom}_{A}\left(U \otimes_{A} U, A\right) \otimes_{A} U \approx \operatorname{Hom}_{A}(U, A) \otimes_{A} \operatorname{Hom}_{A}(U, A) \otimes_{A} U \approx \operatorname{Hom}_{A}(U, A)$ $\otimes_{A} A$, there exist $f_{i}$ in $\operatorname{Hom}_{A}(U, A)$ and $a_{i}$ in $A, i=1,2, \cdots n$ such that $B(x, y)=\sum_{i=1}^{n} f_{i}(x) a_{i} y$ for all $x$ and $y$ in $U$. Put $f=\sum_{i=1}^{n} a_{i} f_{i}$ in $\operatorname{Hom}_{A}(U, A)$, then we have $q^{\prime}(x)=B(x, x)=f(x) x$ for all $x$ in $U$.

Leinma 2. Let $U$ be a finitely generated projective A-module of rank 1, and $f$ and $g$ elements in $\operatorname{Hom}_{A}(U, A)$. If $f(x) x=g(x) x$ for all $x$ in $U$, then $f=g$.

Proof. If $f(x) x=g(x) x$ for all $x$ in $U$, then we have also $f \otimes I(x) x=g \otimes I(x) x$ for all $x$ in $U_{\mathfrak{m}}=U \otimes A_{\mathrm{m}}$ and for every maximal ideal $\mathfrak{m}$ of $A$. For the local ring $A$, this lemma is clear, therefore we get easily $f=g$.

1) cf. p. 490 in [5]. 
Thus, for a given quadratic extension $B$ of $A$ there exist a finitely generated projective $A$-module $U$ of rank 1, an $A$-homomorphism $f: U \rightarrow A$ and a quadratic form $q: U \rightarrow A$ such that $B=A \oplus U$ and $x^{2}=f(x) x+q(x)$ for all $x$ in $U$. Conversely, if a finitely generated projective $A$-module $U$ of rank 1, $A$-homomorphism $f: U \rightarrow A$ and a quadratic form $q: U \rightarrow A$ are given, then a quadratic extension $B=A \oplus U$ of $A$ is constracted by $x^{2}=f(x) x+q(x)$ for $x$ in $U$. We denote such a quadratic extension of $A$ by $B=(U, f, q)$.

In general, we can define as follows:

DEFINITION. Let $P$ be a finitely generated projective and faithful $A$-module, $f: P \rightarrow A$ an $A$-homomorphism and $q: P \rightarrow A$ a quadratic form. Let $T(P)=A \oplus P \oplus P \otimes_{A} P \oplus \ldots$ be the tensor algebra of $P$ over $A$. We denote by $(P, f, q)$ the residue $\operatorname{ring} T(P) /(x \otimes x-f(x) x-q(x) ; x \in P)$ of $T(P)$ by the ideal generated from the set $\{x \otimes x-f(x) x-q(x) ; x \in P\}{ }^{2)}$

Proposition 1. Let $(U, f, q)$ and $\left(U^{\prime}, f^{\prime}, q^{\prime}\right)$ be quadratic extensions of $A$. Then $(U, f, q)$ and $\left(U^{\prime}, f^{\prime}, q^{\prime}\right)$ are A-algebra-isomorphic if and only if there exist an $A$-isomorphism $\sigma_{1}: U \rightarrow U^{\prime}$ and an A-homomorphism $g: U$ $\rightarrow A$ satisfying the following identities;

$$
\begin{aligned}
& q^{\prime} \circ \sigma_{1}=f g+q-g^{2} \\
& f^{\prime} \circ \sigma_{1}=f-2 g,
\end{aligned}
$$

where $f g, g^{2}$ and $q^{\prime} \circ \sigma_{1}$ are defined by $f g(x)=f(x) g(x), g^{2}(x)=g(x)^{2}$ and $q^{\prime} \circ \sigma_{1}(x)=q^{\prime}\left(\sigma_{1}(x)\right)$ for $x$ in $U$.

Proof. Let $\sigma:(U, f, q)=A \oplus U \rightarrow\left(U^{\prime}, f^{\prime}, q^{\prime}\right)=A \oplus U^{\prime}$ be an $A$ algebra-isomorphism. Then there exist an $A$-isomorphism $\sigma_{1}: U \rightarrow U^{\prime}$ and an $A$-homomorphism $g: U \rightarrow A$ such that $\sigma(x)=g(x)+\sigma_{1}(x)$ for $x$ in $U$. Since $\sigma$ satisfies $\sigma\left(x^{2}\right)=\sigma(x)^{2}$ for $x$ in $U$, we get the following identity

$$
f(x) g(x)+q(x)+f(x) \sigma_{1}(x)=g(x)^{2}+q^{\prime}\left(\sigma_{1}(x)\right)+\left(f^{\prime}\left(\sigma_{1}(x)\right)+2 g(x)\right) \sigma_{1}(x)
$$

for all $x$ in $U$. Therefore we have

$$
\begin{aligned}
& f(x) g(x)+q(x)=g(x)^{2}+q^{\prime}\left(\sigma_{1}(x)\right) \\
& f(x) \sigma_{1}(x)=\left(f^{\prime}\left(\sigma_{1}(x)\right)+2 g(x)\right) \sigma_{1}(x)
\end{aligned}
$$

2) The composition of natural homomorphisms $A \oplus U \subset \longrightarrow T(U) \rightarrow T(U) /(x \otimes x-$ $f(x) x-q(x) ; x \in U)$ is an $A$-isomorphism as $A$-modules. For any quadratic extension $C=A \oplus U$ satisfying $x^{2}=f(x) x+q(x)$ for all $x \in U, C \approx T(U) /(x \otimes x-f(x) x-q(x) ; x \in U)$ as $A$-algebras. 
for all $x$ in $U$. From (2) we have $f(x) x=\left(f^{\prime}\left(\sigma_{1}(x)\right)+2 g(x)\right) x$ for $x$ in $U$, and by Lemma 2, we get $f(x)=f^{\prime}\left(\sigma_{1}(x)\right)+2 g(x)$ for $x$ in $U$. Thus, we have the identities of this proposition. The converse is obvious.

LEMMA 3. Let $\left(U_{i}, f_{i}, q_{i}\right)$ and $\left(U_{i}^{\prime}, f_{i}^{\prime}, q_{i}^{\prime}\right)$ be A-algebra-isomorphic quadratic extensions of $A, i=1,2$. Then $\left(U_{1} \otimes_{A} U_{2}, f_{1} \otimes f_{2}, f_{1}^{2} \bar{\otimes} q_{2}+q_{1} \bar{\otimes} f_{2}^{2}\right.$ $\left.+2 q_{1} \otimes q_{2}\right)$ and $\left(U_{1}^{\prime} \otimes_{A} U_{2}^{\prime}, f_{1}^{\prime} \otimes f_{2}^{\prime}, f_{1}^{\prime 2} \bar{\otimes} q_{2}^{\prime}+q_{1}^{\prime} \otimes f_{2}^{\prime 2}+2 q_{1}^{\prime} \otimes q_{2}^{\prime}\right)$ are also A-algebra-isomorphic, where $f_{1}^{2} \bar{\otimes} q_{2}+q_{1} \bar{\otimes} f_{2}^{2}+2 q_{1} \otimes q_{2}(x \otimes y)=f_{1}(x)^{2} q_{2}(y)$ $+q_{1}(x) f_{2}(y)^{2}+4 q_{1}(x) q_{2}(y), f_{1}^{2} \bar{\otimes} q_{2}+q_{1} \bar{\otimes} f_{2}^{2}+2 q_{1} \otimes q_{2}\left(\sum_{i=1}^{n} x_{i} \otimes y_{i}\right)=$ $\sum_{i=1}^{n}\left(f_{1}\left(x_{i}\right)^{2} q_{2}\left(y_{i}\right)+q_{1}\left(x_{i}\right) f_{2}\left(y_{i}\right)^{2}+4 q_{1}\left(x_{i}\right) q_{2}\left(y_{i}\right)\right)+\sum_{i<j}^{n}\left(f_{1}\left(x_{i}\right) f_{1}\left(x_{j}\right) B_{q_{2}}\left(y_{i}, y_{j}\right)\right.$ $\left.+B_{q_{1}}\left(x_{i}, x_{j}\right) f_{2}\left(y_{i}\right) f_{2}\left(y_{j}\right)+2 B_{q_{1}}\left(x_{i}, x_{j}\right) B_{q_{2}}\left(y_{i}, y_{j}\right)\right), \quad(n>1)$, and $f_{1} \otimes f_{2}$ $\left(\sum_{i=1}^{n} x_{i} \otimes y_{i}\right)=\sum_{i=1}^{n} f_{1}\left(x_{i}\right) f_{2}\left(y_{i}\right)$ for $\sum_{i=1}^{n} x_{i} \otimes y_{i}$ and $x \otimes y$ in $U_{1} \otimes{ }_{A} U_{2}$, (cf. (2.8) in [5]). ${ }^{3)}$

Proof. By Proposition 1, there exist $A$-isomorphisms $\sigma_{1}: U_{1} \rightarrow U_{1}^{\prime}$ and $\sigma_{2}: U_{2} \rightarrow U_{2}^{\prime}$, and $A$-homomorphisms $g_{1}: U_{1} \rightarrow A$ and $g_{2}: U_{2} \rightarrow A$ such that $q_{1}^{\prime} \circ \sigma_{1}=f_{1} g_{1}+q_{1}-g_{1}{ }^{2}, f_{1}^{\prime} \circ \sigma_{1}=f_{1}-2 g_{1}$, and $q_{2}^{\prime} \circ \sigma_{2}=f_{2} g_{2}+q_{2}-g_{2}{ }^{2}$, $f_{2}^{\prime} \circ \sigma_{2}=f_{2}-2 g_{2}$. By the computation, we get the following:

For any element $x \otimes y$ in $U_{1} \otimes_{A} U_{2},\left(f_{1}^{\prime 2} \bar{\otimes} q_{2}^{\prime}+q_{1}^{\prime} \bar{\otimes} f_{2}^{\prime 2}+2 q_{1}^{\prime} \otimes q_{2}^{\prime}\right) \circ\left(\sigma_{1} \otimes \sigma_{2}\right)$ $(x \otimes y)=\left(f_{1}(x)-2 g_{1}(x)\right)^{2}\left(f_{2}(y) g_{2}(y)+q_{2}(y)-g_{2}(y)^{2}\right)+\left(f_{1}(x) g_{1}(x)+q_{1}(x)-\right.$ $\left.g_{1}(x)^{2}\right)\left(f_{2}(y)-2 g_{2}(y)\right)^{2}+4\left(f_{1}(x) g_{1}(x)+q_{1}(x)-g_{1}(x)^{2}\right)\left(f_{2}(y) g_{2}(y)+q_{2}(y)-\right.$ $\left.g_{2}(y)^{2}\right)=f_{1}(x) f_{2}(y)\left(f_{1}(x) g_{2}(y)+g_{1}(x) f_{2}(y)-2 g_{1}(x) g_{2}(y)\right)+\left(f_{1}(x)^{2} q_{2}(y)+\right.$ $\left.q_{1}(x) f_{2}(y)^{2}+4 q_{1}(x) q_{2}(y)\right)-\left(f_{1}(x) g_{2}(y)+g_{1}(x) f_{2}(y)-2 g_{1}(x) g_{2}(y)\right)^{2}=\left[\left(f_{1} \otimes f_{2}\right)\right.$ $\left(f_{1} \otimes g_{2}+g_{1} \otimes f_{2}-2 g_{1} \otimes g_{2}\right)+\left(f_{1}^{2} \bar{\otimes} q_{2}+q_{1} \otimes f_{2}^{2}+2 q_{1} \otimes q_{2}\right)-\left(f_{1} \otimes g_{2}+g_{1} \otimes f_{2}\right.$ $\left.\left.-2 g_{1} \otimes g_{2}\right)^{2}\right](x \otimes y)$. Using the identities

$$
B_{q_{1}}\left(\sigma_{1}\left(x_{i}\right), \sigma_{1}\left(x_{j}\right)\right)=f_{1}\left(x_{i}\right) g_{1}\left(x_{j}\right)+f_{1}\left(x_{j}\right) g_{1}\left(x_{i}\right)+B_{q_{1}}\left(x_{i}, x_{j}\right)-2 g_{1}\left(x_{i}\right) g_{1}\left(x_{j}\right)
$$

and $B_{q_{2}^{\prime}}\left(\sigma_{2}\left(y_{i}\right), \sigma_{2}\left(y_{j}\right)\right)=f_{2}\left(y_{i}\right) g_{2}\left(y_{j}\right)+f_{2}\left(y_{j}\right) g_{2}\left(y_{i}\right)+B_{q_{2}}\left(y_{i}, y_{j}\right)-2 g_{2}\left(y_{i}\right) g_{2}\left(y_{j}\right)$ for $x_{i} \otimes y_{i}$ and $x_{j} \otimes y_{j}$ in $U_{1} \otimes{ }_{A} U_{2}$, we get as follows; $f_{1}^{\prime}\left(\sigma_{1}\left(x_{i}\right)\right) f_{1}^{\prime}\left(\sigma_{1}\left(x_{j}\right)\right)$ $B_{q_{2}^{\prime}}\left(\sigma_{2}\left(y_{i}\right), \sigma_{2}\left(y_{j}\right)\right)+B_{q_{1}^{\prime}}\left(\sigma_{1}\left(x_{i}\right), \sigma_{1}\left(x_{j}\right)\right) f_{2}^{\prime}\left(\sigma_{2}\left(y_{i}\right)\right) f_{2}^{\prime}\left(\sigma_{2}\left(y_{j}\right)\right)+2 B_{q_{1}^{\prime}}\left(\sigma_{1}\left(x_{i}\right), \sigma_{1}\left(x_{j}\right)\right)$ $B_{q_{2}^{\prime}}\left(\sigma_{2}\left(y_{i}\right), \sigma_{2}\left(y_{j}\right)\right)=\left(f_{1}\left(x_{i}\right)-2 g_{1}\left(x_{i}\right)\right)\left(f_{1}\left(x_{j}\right)-2 g_{1}\left(x_{j}\right)\right)\left(f_{2}\left(y_{i}\right) g_{2}\left(y_{j}\right)+f_{2}\left(y_{j}\right) g_{2}\left(y_{i}\right)\right.$ $\left.+B_{q_{2}}\left(y_{i}, y_{j}\right)-2 g_{2}\left(y_{i}\right) g_{2}\left(y_{j}\right)\right)+\left(f_{1}\left(x_{i}\right) g_{1}\left(x_{j}\right)+f_{1}\left(x_{j}\right) g_{1}\left(x_{i}\right)+B_{q_{1}}\left(x_{i}, x_{j}\right)-\right.$ $\left.2 g_{1}\left(x_{i}\right) g_{1}\left(x_{j}\right)\right)\left(f_{2}\left(y_{i}\right)-2 g_{2}\left(y_{i}\right)\right)\left(f_{2}\left(y_{j}\right)-2 g_{2}\left(y_{j}\right)\right)+2\left(f_{1}\left(x_{i}\right) g_{1}\left(x_{j}\right)+f_{1}\left(x_{j}\right) g_{1}\left(x_{i}\right)\right.$ $\left.+B_{q_{1}}\left(x_{i}, x_{j}\right)-2 g_{1}\left(x_{i}\right) g_{1}\left(x_{j}\right)\right)\left(f_{2}\left(y_{i}\right) g_{2}\left(y_{j}\right)+f_{2}\left(y_{j}\right) g_{2}\left(y_{i}\right)+B_{q_{2}}\left(y_{i}, y_{j}\right)-2 g_{2}\left(y_{i}\right)\right.$ $\left.g_{2}\left(y_{j}\right)\right)=f_{1}\left(x_{i}\right) f_{2}\left(y_{i}\right)\left(f_{1}\left(x_{j}\right) g_{2}\left(y_{j}\right)+g_{1}\left(x_{j}\right) f_{2}\left(y_{j}\right)-2 g_{1}\left(x_{j}\right) g_{2}\left(y_{j}\right)\right)+f_{1}\left(x_{j}\right) f_{2}\left(y_{j}\right)$ $\left(f_{1}\left(x_{i}\right) g_{2}\left(y_{i}\right)+g_{1}\left(x_{i}\right) f_{2}\left(y_{i}\right)-2 g_{1}\left(x_{i}\right) g_{2}\left(y_{i}\right)\right)+f_{1}\left(x_{i}\right) f_{1}\left(x_{j}\right) B_{q_{2}}\left(y_{i}, y_{j}\right)+B_{q_{1}}\left(x_{i}, x_{j}\right)$

3) $f^{2} \bar{\otimes} q^{\prime}$ and $f^{2} \otimes q^{\prime}$ are defined by $f^{2} \bar{\otimes} q^{\prime}\left(\sum x_{i} \otimes y_{i}\right)=\sum_{i} f\left(x_{i}\right)^{2} q^{\prime}\left(y_{i}\right)+\sum_{i<j} \dot{f}\left(x_{i}\right) f\left(x_{j}\right)$ $B_{q^{\prime}}\left(y_{i}, y_{j}\right)$ and $f^{2} \otimes q^{\prime}\left(\sum x_{i} \otimes y_{i}\right)=\sum_{i} 2 f\left(x_{i}\right)^{2} q^{\prime}\left(y_{i}\right)+\sum_{i<j} B_{f^{2}}\left(x_{i}, x_{j}\right) B_{q^{\prime}}\left(y_{i}, y_{j}\right)$ for $\sum x_{i} \otimes y_{i}$ in $M \otimes_{A} M^{\prime}$. 
$f_{2}\left(y_{i}\right) f_{2}\left(y_{j}\right)+2 B_{q_{1}}\left(x_{i}, x_{j}\right) B_{q_{2}}\left(y_{i}, y_{j}\right)-2\left(f_{1}\left(x_{i}\right) g_{2}\left(y_{i}\right)+g_{1}\left(x_{i}\right) f_{2}\left(y_{i}\right)-2 g_{1}\left(x_{i}\right)\right.$ $\left.g_{2}\left(y_{i}\right)\right)\left(g_{1}\left(x_{j}\right) f_{2}\left(y_{j}\right)+f_{1}\left(x_{j}\right) g_{2}\left(y_{j}\right)-2 g_{1}\left(x_{j}\right) g_{2}\left(y_{j}\right)\right)$. Accordingly, we get $\left(f_{i}^{\prime \prime} \otimes q_{2}^{\prime}+q_{1}^{\prime} \otimes f_{2}^{\prime 2}+2 q_{1}^{\prime} \otimes q_{2}^{\prime}\right) \circ\left(\sigma_{1} \otimes \sigma_{2}\right)\left(\sum_{i=1}^{n} x_{i} \otimes y_{i}\right)=\left[\left(f_{1} \otimes f_{2}\right)\left(f_{1} \otimes g_{2}+\right.\right.$ $\left.\left.g_{1} \otimes f_{2}-2 g_{1} \otimes g_{2}\right)+\left(f_{1}^{2} \bar{\otimes} q_{2}+q_{1} \bar{\otimes} f_{2}^{2}+2 q_{1} \otimes q_{2}\right)+\left(f_{1} \otimes g_{2}-2 g_{1} \otimes g_{2}\right)^{2}\right]$ $\left(\sum_{i=1}^{n} x_{i} \otimes y_{i}\right)$ for all $\sum_{i=1}^{n} x_{i} \otimes y_{i}$ in $U_{1} \otimes_{A} U_{2}$. Put $G=f_{1} \otimes g_{2}+g_{1} \otimes f_{2}-$ $2 g_{1} \otimes g_{2}$, then $G$ is an $A$-homomorphism of $U_{1} \otimes_{A} U_{2}$ to $A$, $\sigma_{1} \otimes \sigma_{2}$ is an $A$-isomorphism of $U_{1} \otimes_{A} U_{2}$ to $U_{1}^{\prime} \otimes_{A} U_{2}^{\prime}$, and these satisfy $\left(f_{1}^{\prime 2} \otimes q_{2}^{\prime}+\right.$ $\left.q_{1}^{\prime} \otimes f_{2}^{\prime 2}+2 q_{1}^{\prime} \otimes q_{2}^{\prime}\right) \circ\left(\sigma_{1} \otimes \sigma_{2}\right)=\left(f_{1} \otimes f_{2}\right) G+\left(f_{1}^{2} \bar{\otimes} q_{2}+q_{1} \bar{\otimes} f_{2}^{2}+2 q_{1} \otimes q_{2}\right)+G^{2}$, and $\left(f_{1}^{\prime} \otimes f_{2}^{\prime}\right) \circ\left(\sigma_{1} \otimes \sigma_{2}\right)=f_{1} \otimes f_{2}-2 G$.

By Proposition 1, we have $\left(U_{1} \otimes_{A} U_{2}, f_{1} \otimes f_{2}, f_{1}^{2} \bar{\otimes} q_{2}+q_{1} \bar{\otimes} f_{2}^{2}+2 q_{1} \otimes q_{2}\right)$ and $\left(U_{1}^{\prime} \otimes_{A} U_{2}^{\prime}, f_{1}^{\prime} \otimes f_{2}^{\prime}, f_{1}^{\prime \prime} \bar{\otimes} q_{2}^{\prime}+q_{1}^{\prime} \bar{\otimes} f_{2}^{\prime 2}+2 q_{1}^{\prime} \otimes q_{2}^{\prime}\right)$ are isomorphic as $A$ algebras.

Definition. We denote by $Q(A)$ the set of all $A$-algebra-isomorphism classes $[U, f, q]$ of quadratic extensions $(U, f, q)$ of $A$.

Proposition 2. $Q(A)$ forms an abelian semi-group with unit element $[A, 1,0]$ by the product $[U, f, q] \cdot\left[U^{\prime}, f^{\prime}, q^{\prime}\right]=\left[U \otimes_{A} U^{\prime}, f \otimes f^{\prime}\right.$, $\left.f^{2} \bar{\otimes} q^{\prime}+q^{\prime} \bar{\otimes} f^{\prime 2}+2 q \otimes q^{\prime}\right]$, where $(A, a, b)$ denotes a quadratic extension $A \oplus A v$ such that $v^{2}=a v+b, a$ and $b$ in $A$, i.e. $f(v)=a, q(v)=b$.

Proof. By Lemma 3, the product in $Q(A)$ is well defined. The associative law is easily seen as follows; $\left([U, f, q]\left[U^{\prime}, f^{\prime}, q^{\prime}\right]\right)\left[U^{\prime \prime}, f^{\prime \prime}, q^{\prime \prime}\right]=$ $\left[U \otimes_{A} U^{\prime} \otimes_{A} U^{\prime \prime}, f \otimes f^{\prime} \otimes f^{\prime \prime}, f^{2} \bar{\otimes} f^{\prime 2} \bar{\otimes} q^{\prime \prime}+f^{2} \bar{\otimes} q^{\prime} \bar{\otimes} f^{\prime \prime 2}+q \bar{\otimes} f^{\prime 2} \bar{\otimes} f^{\prime \prime 2}+\right.$ $\left.2\left(q \otimes q^{\prime} \otimes f^{\prime \prime 2}+q \bar{\otimes} f^{\prime 2} \otimes q^{\prime \prime}+f^{2} \bar{\otimes} q^{\prime} \otimes q^{\prime \prime}\right)+4 q \otimes q^{\prime} \otimes q^{\prime \prime}\right]=[U, f, q]$ $\left(\left[U^{\prime}, f^{\prime}, q^{\prime}\right]\left[U^{\prime \prime}, f^{\prime \prime}, q^{\prime \prime}\right]\right) .^{4)}$

Definition. Let $P$ be a finitely generated projective and faithful $A$-module, $f: P \rightarrow A$ an $A$-homomorphism and $q: P \rightarrow A$ a quadratic form. For the $A$-algebra $(P, f, q)=T(P) /(x \otimes x-f(x) x-q(x) ; x \in P)$, we consider a symmetric bilinear form $D_{f, q}: P \times P \rightarrow A$ defined by $D_{f, q}(x, y)=$ $f(x) f(y)+2 B_{q}(x, y)$ for $x, y$ in $P$, where $B_{q}(x, y)=q(x+y)-q(x)-q(y)$ for $x, y$ in $P$. Then we shall call the biliriear $A$-module $\left(P, D_{f, q}\right)$ the discriminant of $(P, f, q)$.

Remark 1. If 2 is inversible in $A$, then we have that $(P, f, q)$ is a separable algebra over $A$ if and only if $\left(P, D_{f, q}\right)$ is a non-degenerate bilinear $A$-module, i.e. $P \rightarrow \operatorname{Hom}_{A}(P, A) ; x \backsim D_{f, q}(x,-)$ is an isomorphism.

4) $\left(q \bar{\otimes} f^{2}\right) \otimes q^{\prime}=q \otimes\left(f^{2} \bar{\otimes} q^{\prime}\right),\left(f^{2} \bar{\otimes} q^{\prime}\right) \otimes q^{\prime \prime}=f^{2} \bar{\otimes}\left(q^{\prime} \otimes q^{\prime \prime}\right)$. 
Proof. $d=f^{2}+4 q$ is a quadratic form of $P$ to $A$, and satisfies $d(x)=f(x)^{2}+2 B_{q}(x, x)=D_{f, q}(x, x)$. In the tensor algebra $T(P)$, we put $P^{\prime}=\{x-(1 / 2) f(x) \in A \oplus P \subset T(P) ; x \in P\}$, then the map $P \rightarrow P^{\prime} ; x \sim x$ -(1/2)f(x) is an $A$-isomorphism. We denote by $h$ the inverse isomorphism of it. For the ideal of $T(P)$ generated by the set $\{x \otimes x-f(x) x$ $-q(x) ; x \in P\}=\left\{x \otimes x-d(h(x / 2)) ; x \in P^{\prime}\right\}$, we have $(P, f, q)=T(P) /(x \otimes x$ $\left.-f(x) x-q(x) ; x \in P)=T\left(P^{\prime}\right) /(x \otimes x-d(h(x) / 2)) ; x \in P^{\prime}\right)=\left(P^{\prime}, 0, d \circ(1 / 2) h\right)$, since $T(P)=T\left(P^{\prime}\right)$. But, $\left(P^{\prime}, 0, d \circ(1 / 2) h\right)$ is a Clifford algebra $\mathrm{Cl}\left(P^{\prime}, d \circ(1 / 2) h\right)$ of a quadratic module $\left(P^{\prime}, d \circ(1 / 2) h\right)$. It is known that $\mathrm{Cl}\left(P^{\prime}, d \circ(1 / 2) h\right)$ is a separable algebra over $A$ if and only if $\left(P^{\prime}, d \circ(1 / 2) h\right)$ is non-degenerated. Since $(P, d)$ and $\left(P^{\prime}, d \circ(1 / 2) h\right)$ are isometric, we get this remark.

THEOREM 1. Let $U$ be a finitely generated projective $A$-module of rank $1, f: U \rightarrow A$ an $A$-homomorphism, $q: U \rightarrow A$ a quadratic form, and $(U, f, q)$ the quadratic extension of $A$. Then the following conditions are equivalent:

1) $(U, f, q)$ is a separable algebra over $A$.

2) $\left(U, D_{f, q}\right)$ is a non-degenerate bilinear $A$-module.

3) $[U, f, q]^{2}=[A, 1,0]$.

Proof. 1) $\rightleftarrows 2$ ): To prove the equivalence of the conditions 1 ) and 2 ), we may assume that $A$ is a local ring. Let $A$ be the local ring. Then $U=A u$ and $(U, f, q) \approx A[X] /\left(X^{2}-a X-b\right)$, where $a=f(u), b=q(u)$. Hence, $(U, f, q)$ is separable over $A$ if and only if $a^{2}+4 b=f^{2}+4 q(u)$ $=D_{f, q}(u, u)$ is inversible in $A$. On the other hand, $\left(U, D_{f, q}\right)$ is nondegenerated if and only if $D_{f, q}(u, u)$ is inversible in $A$. Therefore, we obtain the equivalence.

$2) \rightarrow 3)$ : Assume that $\left(U, D_{f, q}\right)$ is non-degenerate. Then the $A$ isomorphism $U \rightarrow \operatorname{Hom}_{A}(U, A) ; x \sim D_{f, q}(x,-)$ induces an $A$-isomorphism $D_{f, q}: U \otimes_{A} U \rightarrow A ; x \otimes y \backsim D_{f, q}(x, y)$. Put $\sigma_{1}=D_{f, q}$ and $g=-B_{q}$. Then we have $I \circ \sigma_{1}=D_{f, q}=f \otimes f+2 B_{q}=f \otimes f-2 g$. Furthermore, we can prove the following identity:

$$
(f \otimes f) g+\left(f^{2} \bar{\otimes} q+q \bar{\otimes} f^{2}+2 q \otimes q\right)-g^{2}=0 .
$$

Because, by the localizations of $A$ and $U$ by every maximal ideal $\mathfrak{m}$ of $A$, we can check that quadratic forms $f^{2} \bar{\otimes} q+q \bar{\otimes} f^{2}-B_{q} \cdot f \otimes f: U \otimes_{A} U$ $\rightarrow A$, and $2 q \otimes q-B_{q}^{2}: U \otimes_{A} U \rightarrow A$ are equal to 0 . Thus, by Proposition 1 we get $[U, f, q]^{2}=\left[U \otimes_{A} U, f \otimes f, f^{2} \bar{\otimes} q+q \bar{\otimes} f^{2}+2 q \otimes q\right]=$ 
$[A, 1,0]$.

$3) \rightarrow 2)$ : Let $[U, f, q]^{2}=[A, 1,0]$. To prove the condition 2) it is sufficient to show that for any maximal ideal $\mathrm{m}$ of $A, D_{f, q}(u, u)$ is inversible in $A_{\mathrm{m}}$, where $U_{\mathrm{m}}=A_{\mathrm{m}} u$. Now, we assume $A$ is a local ring with maximal ideal $\mathfrak{m}$ and $U=A u$. We shall show $D_{f, q}(u, u)=f(u)^{2}+$ $2 B_{q}(u, u)=f(u)^{2}+4 q(u) \notin \mathfrak{m}$. From $[U, f, q]^{2}=[A, 1,0]$, there exist an $A$-homomorphism $g: U \otimes_{A} U \rightarrow A$ and an $A$-isomorphism $\sigma_{1}: U \otimes_{A} U \rightarrow A$ such that $\sigma_{1}(x \otimes y)=f(x) f(y)-2 g(x \otimes y)$ and $0=f(x) f(y) g(x \otimes y)+$ $f(x)^{2} q(y)+q(x) f(y)^{2}+4 q(x) q(y)+g(x \otimes y)^{2}$ for all $x \otimes y \in U \otimes_{A} U$. Especially, taking $x=y=u$, we get

$$
\sigma_{1}(u \otimes u)=f(u)^{2}-2 g(u \otimes u)
$$

and

$$
0=f(u)^{2} g(u \otimes u)+2 f(u)^{2} q(u)+4 q(u)^{2}-g(u \otimes u)
$$

Eliminating $f(u)^{2}$ from (3) and (4), we get $\left(\sigma_{1}(u \otimes u)+2 g(u \otimes u)\right) g(u \otimes u)$ $+2\left(\sigma_{1}(u \otimes u)+2 g(u \otimes u)\right) q(u)+4 q(u)^{2}-g(u \otimes u)^{2}=0$, and so

$$
\left(\sigma_{1}(u \otimes u)+g(u \otimes u)+2 q(u)\right)(g(u \otimes u)+2 q(u))=0 .
$$

If $g(u \otimes u)+2 q(u)$ is contained in $\mathfrak{m}$, then from $\sigma_{1}(u \otimes u) \notin \mathfrak{m}, \sigma_{1}(u \otimes u)$ $+g(u \otimes u)+2 q(u)$ is inversible in $A$. Therefore, we have $g(u \otimes u)+$ $2 q(u)=0$, and $D_{f, q}(u, u)=f(u)^{2}+4 q(u)=f(u)^{2}-2 g(u \otimes u)=\sigma_{1}(u \otimes u)$ is inversible in $A$. If $g(u \otimes u)+2 q(u) \notin \mathfrak{m}$, then $\sigma_{1}(u \otimes u)+g(u \otimes u)+$ $2 q(u)=0$. From (3) and $2 \sigma_{1}(u \otimes u)+2 g(u \otimes u)+4 q(u)=0$, we get $\sigma_{1}(u \otimes u)+f(u)^{2}+4 q(u)=0$, accordingly, $D_{f, q}(u, u)=f(u)^{2}+4 q(u)=$ $-\sigma_{1}(u \otimes u)$ is inversible in $A$.

COROLlary 1. The set $Q_{s}(A)$ of A-algebra-isomorphism classes of the separable quadratic extensions of $A$ forms an abelian group with exponent 2.

Proposition 3. Let $(U, f, q)$ be a quadratic extension of $A$. The $\operatorname{map} \tau_{f}:(U, f, q) \rightarrow(U, f, q) ; a+x \backsim a+f(x)-x$ is an A-algebra-isomorphism such that $\tau_{f}{ }^{2}=I$. If $(U, f, q)$ and $\left(U^{\prime}, f^{\prime}, q^{\prime}\right)$ are quadratic extensions of $A$ and $\sigma:(U, f, q) \rightarrow\left(U^{\prime}, f^{\prime}, q^{\prime}\right)$ is an A-algebra-isomorphim, then we have the following commutative diagram; 


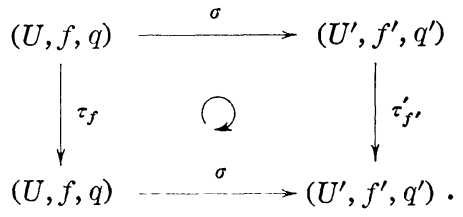

Proof. From Proposition 1, there exist $g$ in $\operatorname{Hom}_{A}(U, A)$ and $A$ isomorphism $\sigma_{1}: U \rightarrow U^{\prime}$ such that $\sigma(x)=g(x)+\sigma_{1}(x)$ and $f^{\prime}\left(\sigma_{1}(x)\right)=f(x)$ $-2 g(x)$ for all $x$ in $U$. Therefore, $\tau_{f^{\prime}}^{\prime}(\sigma(x))=g(x)+\tau_{f^{\prime}}^{\prime}\left(\sigma_{1}(x)\right)=g(x)+$ $f^{\prime}\left(\sigma_{1}(x)\right)-\sigma_{1}(x)=g(x)+f(x)-2 g(x)-\sigma_{1}(x)=f(x)-\left(g(x)+\sigma_{1}(x)\right)=$ $f(x)-\sigma(x)=\sigma(f(x)-x)=\sigma\left(\tau_{f}(x)\right)$, for all $x$ in $U$.

Remark 2.

1) In Proposition 3, if we take $\sigma=I$, then $\tau_{f}=\tau_{f^{\prime}}^{\prime}$.

2) If $(U, f, q)$ is a separable algebra over $A$, then $\tau_{f}$ is the unique $A$-algebra-automorphism of $(U, f, q)$ which is not the identity.

Let $B=(U, f, q)$ and $B^{\prime}=\left(U^{\prime}, f^{\prime}, q^{\prime}\right)$ be separable quadratic extensions of $A$. Then $G=\left\{\tau_{f}, I\right\}$ and $G^{\prime}=\left\{\tau_{f^{\prime}}^{\prime}, I\right\}$ are the groups of automorphisms of $B$ over $A$ and $B^{\prime}$ over $A$, respectively. In [1], [3] and [4], the product $B * B^{\prime}$ of quadratic extensions $B$ and $B^{\prime}$ was defined as the fixed subalgebra $\left(B \otimes{ }_{A} B^{\prime}\right)^{\tau_{f} \otimes \tau_{f^{\prime}}}=\left\{x \in B \otimes_{A} B^{\prime} ; \tau_{f} \otimes \tau_{f}^{\prime}(x)=x\right\}$ of $B \otimes_{A} B^{\prime}$ by $\tau_{f} \otimes \tau_{f^{\prime}}^{\prime}$. But this product coincides with our one.

Proposition 4. Let $(U, f, q)$ and $\left(U^{\prime}, f^{\prime}, q^{\prime}\right)$ be separable quadratic extensions of $A$. Then we have $\left[(U, f, q) \otimes_{A}\left(U^{\prime}, f^{\prime}, q^{\prime}\right)^{\tau_{f} \otimes \tau_{f^{\prime}}}\right]=[U, f, q]$. $\left[U^{\prime}, f^{\prime}, q^{\prime}\right]$ in $Q_{s}(A)$.

Proof. For $B=(U, f, q)$ and $B^{\prime}=\left(U^{\prime}, f^{\prime}, q^{\prime}\right), B \otimes_{A} B^{\prime}$ is expressed as a direct sum $B \otimes_{A} B^{\prime}=A \oplus U \oplus U^{\prime} \oplus U \otimes_{A} U^{\prime}$. Put $V=\left\{\sum_{i} f\left(x_{i}\right) y_{i}+\right.$ $f^{\prime}\left(y_{i}\right) x_{i}-2 x_{i} \otimes y_{i} \in U \oplus U^{\prime} \oplus U \otimes_{A} U^{\prime}$; for all $\sum_{i} x_{i} \otimes y_{i}$ in $\left.U \otimes_{A} U^{\prime}\right\}$. Then $V$ is an $A$-submodule of $B \otimes_{A} B^{\prime}$, which is $A$-isomorphic to $U \otimes_{A} U^{\prime}$ by the isomorphism $\theta: U \otimes_{A} U^{\prime} \rightarrow V ; x \otimes y \sim f(x) y+f^{\prime}(y) x-2 x \otimes y$. It is easily seen that the $A$-submodule $C=A \oplus V$ of $B \otimes_{A} B^{\prime}$ generated by

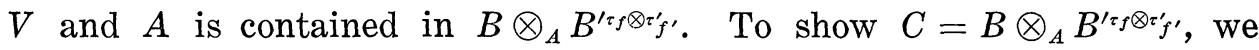
shall prove first that the map $\theta^{\prime}:\left(U \otimes_{A} U^{\prime}, f \otimes f^{\prime}, f^{2} \bar{\otimes} q^{\prime}+q \bar{\otimes} f^{\prime 2}+\right.$ $\left.2 q \otimes q^{\prime}\right)=A \oplus U \otimes_{A} U^{\prime} \rightarrow C=A \oplus V ; a+x \otimes y \sim a+\theta(x \otimes y)$ is an $A$ algebra-isomorphism. We can easily compute that for any $x \otimes y$ in $U \otimes_{A} U^{\prime}, \theta^{\prime}(x \otimes y)^{2}=\left(f(x) y+f^{\prime}(y) x-2 x \otimes y\right)^{2}=f(x)^{2} y^{2}+f^{\prime}(y)^{2} x^{2}+$ $4 x^{2} \otimes y^{2}+2 f(x) f^{\prime}(y) x \otimes y-4 f(x) x \otimes y^{2}-4 f^{\prime}(y) x^{2} \otimes y=f(x)^{2}\left(f^{\prime}(y) y+\right.$ $\left.q^{\prime}(y)\right)+f^{\prime}(y)^{2}(f(x) x+q(x))+4(f(x)+q(x)) \otimes\left(f^{\prime}(y) y+q^{\prime}(y)\right)+2 f(x) f^{\prime}(y)$ 
$x \otimes y-4 f(x) x \otimes\left(f^{\prime}(y) y+q^{\prime}(y)\right)-4 f^{\prime}(y)(f(x) x+q(x)) \otimes y=f(x) f^{\prime}(y)(f(x) y$ $\left.+f^{\prime}(y) x-2 x \otimes y\right)+f(x)^{2} q^{\prime}(y)+f^{\prime}(y)^{2} q(x)+4 q(x) q^{\prime}(y)=f \otimes f^{\prime}(x \otimes y) \theta^{\prime}$ $(x \otimes y)+\left(f^{2} \otimes q^{\prime}+q \otimes f^{\prime 2}+2 q \otimes q^{\prime}\right)(x \otimes y)=\theta^{\prime}\left[\left(f \otimes f^{\prime}(x \otimes y) x \otimes y+\right.\right.$ $\left.\left(f^{2} \bar{\otimes} q^{\prime}+q \bar{\otimes} f^{\prime 2}+q \otimes q^{\prime}\right)(x \otimes y)\right]=\theta^{\prime}\left((x \otimes y)^{2}\right)$, and for $x_{i} \otimes y_{i}, x_{j} \otimes y_{j}$ in $U \otimes_{A} U^{\prime}, 2 \theta^{\prime}\left(x_{i} \otimes y_{i}\right) \cdot \theta^{\prime}\left(x_{j} \otimes y_{j}\right)=2\left(f\left(x_{i}\right) y_{i}+f^{\prime}\left(y_{i}\right) x_{i}-2 x_{i} \otimes y_{i}\right)\left(f\left(x_{j}\right) y_{j}+\right.$ $\left.f^{\prime}\left(y_{j}\right) x_{j}-2 x_{j} \otimes y_{j}\right)=f\left(x_{i}\right) f^{\prime}\left(y_{i}\right)\left(f\left(x_{j}\right) y_{j}+f^{\prime}\left(y_{j}\right) x_{j}-2 x_{j} \otimes y_{j}\right)+f\left(x_{j}\right) f^{\prime}\left(y_{j}\right)$ $\left(f\left(x_{i}\right) y_{i}+f^{\prime}\left(y_{i}\right) x_{i}-2 x_{i} \otimes y_{i}\right)+f\left(x_{i}\right) f\left(x_{j}\right) B_{q^{\prime}}\left(y_{i}, y_{j}\right)+f^{\prime}\left(y_{i}\right) f^{\prime}\left(y_{j}\right) B_{q}\left(x_{i}, x_{j}\right)+$ $2 B_{q}\left(x_{i}, x_{j}\right) B_{q^{\prime}}\left(y_{i}, y_{j}\right)=\theta^{\prime}\left(f\left(x_{i}\right) f^{\prime}\left(y_{i}\right) x_{j} \otimes y_{j}+f\left(x_{j}\right) f^{\prime}\left(y_{j}\right) x_{i} \otimes y_{i}\right)+f\left(x_{i}\right) f\left(x_{j}\right)$ $B_{q^{\prime}}\left(y_{i}, y_{j}\right)+f^{\prime}\left(y_{i}\right) f^{\prime}\left(y_{j}\right) B_{q}\left(x_{i}, x_{j}\right)+2 B_{q}\left(x_{i}, x_{j}\right) B_{q^{\prime}}\left(y_{i}, y_{j}\right)$.

Therefore, we have $\theta^{\prime}\left(\sum_{i} x_{i} \otimes y_{i}\right)^{2}=\theta^{\prime}\left(\left(\sum_{i} x_{i} \otimes y_{i}\right)^{2}\right)$ for any $\sum_{i} x_{i} \otimes y_{i}$ in $U \otimes_{A} U^{\prime}$. Accordingly, $\theta^{\prime}$ is an $A$-algebra isomorphism. Thus, $C$ is also a separable algebra over $A$. Since $B \otimes_{A} B^{\prime}$ is a finitely generated projective $A$-module, $B \otimes_{A} B^{\prime}$ is also finitely generated projective over $C$. Therefore, $C$ is a direct summand of $B \otimes_{A} B^{\prime}$, and hence also a direct summand of $B \otimes_{A} B^{\tau_{f} \otimes \tau_{f^{\prime}}}$ as $C$-module. But, $\operatorname{rank}(C: A)=$ $\operatorname{rank}\left(B \otimes_{A} B^{\prime \tau_{f} \otimes \tau_{f^{\prime}}}: A\right)=2$, hence we have $B \otimes_{A} B^{\prime \tau_{f} \otimes \tau_{f^{\prime}}^{\prime}}=C=A \oplus V \approx$ $\left(U \otimes_{A} U^{\prime}, f \otimes f^{\prime}, f^{2} \otimes q^{\prime}+q \bar{\otimes} f^{\prime 2}+2 q \otimes q^{\prime}\right)$ as $A$-algebra.

\section{Extended quadratic module.}

In this section, we give a generalization of quadratic module. Let $A$ be an arbitrary commutative ring with unit element. Let $M$ be an $A$-module, $f: M \rightarrow A$ an $A$-homomorphism, and $q: M \rightarrow A$ a quadratic form. Then, we call the triple $\langle M, f, q\rangle$ an extended quadratic module

Definition. Lat $\langle M, f, q\rangle$ and $\left\langle M^{\prime}, f^{\prime}, q^{\prime}\right\rangle$ be extended quadratic modules. If there exist an $A$-isomorphism $\sigma: M \rightarrow M^{\prime}$ and $A$-homomorphism $g: M \rightarrow A$ satisfying $q^{\prime} \circ \sigma=q+2 f g-2 g^{2}$ and $f^{\prime} \circ \sigma=f-2 g$, then we call that $\langle M, f, q\rangle$ and $\left\langle M^{\prime}, f^{\prime}, q^{\prime}\right\rangle$ are $A$-isomorphic, and denote by $(\sigma, g):\langle M, f, q\rangle \rightarrow\left\langle M^{\prime}, f^{\prime}, q^{\prime}\right\rangle$ the $A$-isomorphism of extended quadratic modules, or simply $\langle M, f, q\rangle \approx\left\langle M^{\prime}, f^{\prime}, q^{\prime}\right\rangle$.

Then we have easily

1) $(I, 0)$ is identity,

2) $\left(\sigma^{\prime}, g^{\prime}\right)(\sigma, g)=\left(\sigma^{\prime} \circ \sigma, g+g^{\prime} \circ \sigma\right)$ and

3) $(\sigma, g)^{-1}=\left(\sigma^{-1},-g \circ \sigma^{-1}\right)$.

Thus, we can consider a category Qua* $(A)$ in which objects are extended quadratic modules and morphisms are $A$-isomorphisms of extended quadratic modules. Then, Qua* $(A)$ includes the category Qua $(A)$ of the 
ordinaly quadratic modules as a sub-category. Because, $(\sigma, g):\langle M, 0, q\rangle$ $\rightarrow\left\langle M^{\prime}, 0, q^{\prime}\right\rangle$ is an $A$-isomorphism in $\mathrm{Qua}^{*}(A)$ if and only if $\sigma:(M, q) \rightarrow$ $\left(M^{\prime}, q^{\prime}\right)$ is an $A$-isomorphism in Qua $(A)$, therefore we may regard as $\langle M, 0, q\rangle=(M, q)$ and $(\sigma, 0)=\sigma$ in Qua $(A)$.

DEFINITION. Let $\langle M, f, q\rangle$ be an extended quadratic module, and let $B_{f, q}: M \times M \rightarrow A$ be a symmetric bilinear form defined by $B_{f, q}(x, y)=$ $f(x) f(y)+B_{q}(x, y)$ for $x$ and $y$ in $M$. Then, we call the bilinear module $\left(M, B_{f, q}\right)$ the associated bilinear module with $\langle M, f, q\rangle$. If $\left(M, B_{f, q}\right)$ is a non-degenerate bilinear module, then $\langle M, f, q\rangle$ is called a non-degenerate extended quadratic module.

Lemma 4. If $(\sigma, g):\langle M, f, q\rangle \rightarrow\left\langle M^{\prime}, f^{\prime}, q^{\prime}\right\rangle$ is an A-isomorphism in Qua* $(A)$, then we have $B_{f^{\prime}, q^{\prime}}(\sigma(x), \sigma(y))=B_{f, q}(x, y)$ for all $x$ and $y$ in $M$, that is, $\sigma:\left(M, B_{f, q}\right) \rightarrow\left(M^{\prime}, B_{f^{\prime}, q^{\prime}}\right)$ is an A-isomorphism of bilinear modules.

Proof. Since the $A$-isomorphism $\sigma: M \rightarrow M^{\prime}$ and the $A$-homomorphism $g: M \rightarrow A$ satisfy $f^{\prime} \circ \sigma=f-2 g$ and $q^{\prime} \circ \sigma=q+2 f g-2 g^{2}$, we have $B_{f^{\prime}, q^{\prime}}(\sigma(x), \sigma(y))=f^{\prime}(\sigma(x)) f^{\prime}(\sigma(y))+B_{q^{\prime}}(\sigma(x), \sigma(y))=(f(x)-2 g(x))(f(y)-$ $2 g(y))+B_{q}(x, y)+2(f(x) g(y)+f(y) g(x))-4 g(x) g(y)=f(x) f(y)+B_{q}(x, y)$ $=B_{f, q}(x, y)$.

Corollary 2. If $\langle M, f, q\rangle \approx\left\langle M^{\prime}, f^{\prime}, q^{\prime}\right\rangle$ and $\langle M, f, q\rangle$ is non-degenerate, then $\left\langle M^{\prime}, f^{\prime}, q^{\prime}\right\rangle$ is also non-degenerate.

Definition. Let $\left\langle M_{1}, f_{1}, q_{1}\right\rangle$ and $\left\langle M_{2}, f_{2}, q_{2}\right\rangle$ be extended quadratic modules. We define the orthogonal sum $\perp$ and the tensor product $\otimes$ of extended quadratic modules as follows:

$$
\begin{aligned}
& \left\langle M_{1}, f_{1}, q_{1}\right\rangle \perp\left\langle M_{2}, f_{2}, q_{2}\right\rangle=\left\langle M_{1} \oplus M_{2}, f_{1} \perp f_{2}, q_{1} \perp q_{2}-f_{1} \times f_{2}\right\rangle \\
& \left\langle M_{1}, f_{1}, q_{1}\right\rangle \otimes\left\langle M_{2}, f_{2}, q_{2}\right\rangle \\
& =\left\langle M_{1} \otimes M_{2}, f_{1} \otimes f_{2}, f_{1}^{2} \bar{\otimes} q_{2}+q_{1} \bar{\otimes} f_{2}^{2}+q_{1} \otimes q_{2}\right\rangle
\end{aligned}
$$

where $f_{1} \perp f_{2}$ is defined by the $A$-homomorphism $M_{1} \oplus M_{2} \rightarrow A ; x_{1} \oplus x_{2}$ $\sim f_{1}\left(x_{1}\right)+f_{2}\left(x_{2}\right)$, and $f_{1} \times f_{2}$ the quadratic form $M_{1} \oplus M_{2} \rightarrow A ; x_{1} \oplus x_{2}$ $\sim f_{1}\left(x_{1}\right) \cdot f_{2}\left(x_{2}\right)$.

LEMmA 5. Let $\left\langle M_{i}, f_{i}, q_{i}\right\rangle$ and $\left\langle M_{i}^{\prime}, f_{i}^{\prime}, q_{i}^{\prime}\right\rangle$ be extended quadratic modules, and $\left(\sigma_{i}, g_{i}\right):\left\langle M_{i}, f_{i}, q_{i}\right\rangle \rightarrow\left\langle M_{i}^{\prime}, f_{i}^{\prime}, q_{i}^{\prime}\right\rangle$ an A-isomorphism in Qua* $(A)$ for $i=1,2$. Then we have the following A-isomorphisms in Qua* $(A)$; 


$$
\begin{gathered}
\left(\sigma_{1} \oplus \sigma_{2}, g_{1} \perp g_{2}\right):\left\langle M_{1}, f_{1}, q_{1}\right\rangle \perp\left\langle M_{2}, f_{2}, q_{2}\right\rangle \rightarrow\left\langle M_{1}^{\prime}, f_{1}^{\prime}, q_{1}^{\prime}\right\rangle \perp\left\langle M_{2}^{\prime}, f_{2}^{\prime}, q_{2}^{\prime}\right\rangle \\
\left(\sigma_{1} \otimes \sigma_{2}, f_{1} \otimes g_{2}+g_{1} \otimes f_{2}-2 g_{1} \otimes g_{2}\right):\left\langle M_{1}, f_{1}, q_{1}\right\rangle \otimes\left\langle M_{2}, f_{2}, q_{2}\right\rangle \\
\rightarrow\left\langle M_{1}^{\prime}, f_{1}^{\prime}, q_{1}^{\prime}\right\rangle \otimes\left\langle M_{2}^{\prime}, f_{2}^{\prime}, q_{2}^{\prime}\right\rangle
\end{gathered}
$$

Proof. The proof of (7). We shall show that $\left(\sigma_{1} \oplus \sigma_{2}, g_{1} \perp g_{2}\right)$ : $\left\langle M_{1} \oplus M_{2}, f_{1} \perp f_{2}, q_{1} \perp q_{2}-f_{1} \times f_{2}\right\rangle \rightarrow\left\langle M_{1}^{\prime} \oplus M_{2}^{\prime}, f_{1}^{\prime} \perp f_{2}^{\prime}, q_{1}^{\prime} \perp q_{2}^{\prime}-f_{1}^{\prime} \times f_{2}^{\prime}\right\rangle$ is an $A$-isomorphism in Qua* $(A)$. For $x_{1} \oplus x_{2}$ in $M_{1} \oplus M_{2}$, we have

$\left(q_{1}^{\prime} \perp q_{2}^{\prime}-f_{1}^{\prime} \times f_{2}^{\prime}\right) \circ\left(\sigma_{1} \oplus \sigma_{2}\right)\left(x_{1} \oplus x_{2}\right)=q_{1}^{\prime}\left(\sigma_{1}\left(x_{1}\right)\right)+q_{2}^{\prime}\left(\sigma_{2}\left(x_{2}\right)\right)-f_{1}^{\prime}\left(\sigma_{1}\left(x_{1}\right)\right) f_{2}^{\prime}\left(\sigma_{2}\left(x_{2}\right)\right)$ $=q_{1}\left(x_{1}\right)+2 f_{1}\left(x_{1}\right) g_{1}\left(x_{1}\right)-2 g_{1}\left(x_{1}\right)^{2}+q_{2}\left(x_{2}\right)+2 f_{2}\left(x_{2}\right) g_{2}\left(x_{2}\right)-2 g_{2}\left(x_{2}\right)^{2}-\left(f_{1}\left(x_{1}\right)-\right.$ $\left.2 g_{1}\left(x_{1}\right)\right)\left(f_{2}\left(x_{2}\right)-2 g_{2}\left(x_{2}\right)\right)=\left(q_{1} \perp q_{2}-f_{1} \times f_{2}\right)\left(x_{1} \oplus x_{2}\right)+2\left(f_{1} \perp f_{2}\right)\left(g_{1} \perp g_{2}\right)$ $\left(x_{1} \oplus x_{2}\right)-2\left(g_{1} \perp g_{2}\right)^{2}\left(x_{1} \oplus x_{2}\right)$, and

$$
\begin{aligned}
\left(f_{1}^{\prime} \perp f_{2}^{\prime}\right) \circ\left(\sigma_{1} \oplus \sigma_{2}\right) & =f_{1}^{\prime} \circ \sigma_{1} \perp f_{2}^{\prime} \circ \sigma_{2}=\left(f_{1}-2 g_{1}\right) \perp\left(f_{2}-2 g_{2}\right) \\
& =\left(f_{1} \perp f_{2}\right)-2\left(g_{1} \perp g_{2}\right) .
\end{aligned}
$$

The proof of (8) is obtained by similar computations the proof of Lemma 3. We omit this proof.

Definition. We denote by $B_{f, q} \perp B_{f^{\prime}, q^{\prime}}$ the associated bilinear form with $\langle M, f, q\rangle \perp\left\langle M^{\prime}, f^{\prime}, q^{\prime}\right\rangle$, and by $B_{f, q} \otimes B_{f^{\prime}, q^{\prime}}$ the associated bilinear form with $\langle M, f, q\rangle \otimes\left\langle M^{\prime}, f^{\prime}, q^{\prime}\right\rangle$, that is, $B_{f, q} \perp B_{f^{\prime}, q^{\prime}}=B_{f \perp f^{\prime},\left(q \perp q^{\prime}\right)-\left(f \times f^{\prime}\right)}$ and $B_{f, q} \otimes B_{f^{\prime}, q^{\prime}}=B_{f \otimes f^{\prime}, f^{2} \otimes q^{\prime}+q \bar{\otimes} f^{\prime 2}+q \otimes q^{\prime}}$.

Proposition 5. The orthogonal sum and the tensor product of extended quadratic modules $\langle M, f, q\rangle$ and $\left\langle M^{\prime}, f^{\prime}, q^{\prime}\right\rangle$ induce the following identities between the associated bilinear modules with them;

$$
\left(M \oplus M^{\prime}, B_{f, q} \perp B_{f^{\prime}, q^{\prime}}\right)=\left(M, B_{f, q}\right) \perp\left(M^{\prime}, B_{f^{\prime}, q^{\prime}}\right)
$$

i.e. $\quad B_{f, q} \perp B_{f^{\prime}, q^{\prime}}\left(x \oplus x^{\prime}, y \oplus y^{\prime}\right)=B_{f, q}(x, y)+B_{f^{\prime}, q^{\prime}}\left(x^{\prime}, y^{\prime}\right)$ for $x \oplus x^{\prime}$ and $y \oplus y^{\prime}$ in $M \oplus M^{\prime}$, and

$$
\left(M \otimes M^{\prime}, B_{f, q} \otimes B_{f^{\prime}, q^{\prime}}\right)=\left(M, B_{f, q}\right) \otimes\left(M^{\prime}, B_{f^{\prime}, q^{\prime}}\right)
$$

i.e. $\quad B_{f, q} \otimes B_{f^{\prime}, q^{\prime}}\left(\sum_{i} x_{i} \otimes x_{i}^{\prime}, \sum_{j} y_{j} \otimes y_{j}^{\prime}\right)=\sum_{i, j} B_{f, q}\left(x_{i}, y_{j}\right) \cdot B_{f^{\prime}, q^{\prime}}\left(x_{i}^{\prime}, y_{j}^{\prime}\right)$ for $\sum_{i} x_{i} \otimes x_{i}^{\prime}$ and $\sum_{j} y_{j} \otimes y_{j}^{\prime}$ in $M \otimes M^{\prime}$.

Proof. The proof of (9): $B_{f, q} \perp B_{f^{\prime}, q^{\prime}}\left(x \oplus x^{\prime}, y \oplus y^{\prime}\right)=\left(f \perp f^{\prime}\left(x \otimes x^{\prime}\right)\right)$ $\left(f \perp f^{\prime}\left(y \oplus y^{\prime}\right)\right)+B_{\left(q \perp q^{\prime}\right)-\left(f \times f^{\prime}\right)}\left(x \oplus x^{\prime}, y \oplus y^{\prime}\right)=\left(f(x)+f^{\prime}\left(x^{\prime}\right)\right)\left(f(y)+f^{\prime}\left(y^{\prime}\right)\right)$ $+B_{q \perp q^{\prime}}\left(x \oplus x^{\prime}, y \oplus y^{\prime}\right)-B_{f \times f^{\prime}}\left(x \oplus x^{\prime}, y \oplus y^{\prime}\right)=f(x) f(y)+f^{\prime}\left(x^{\prime}\right) f^{\prime}\left(y^{\prime}\right)+$ $f^{\prime}\left(x^{\prime}\right) f(y)+f(x) f^{\prime}\left(y^{\prime}\right)+B_{q}(x, y)+B_{q^{\prime}}\left(x^{\prime}, y^{\prime}\right)-\left(f(x) f^{\prime}\left(y^{\prime}\right)+f(y) f^{\prime}\left(x^{\prime}\right)\right)=$ $B_{f, q}(x, y)+B_{f^{\prime}, q^{\prime}}\left(x^{\prime}, y^{\prime}\right)$, for any $x \oplus x^{\prime}$ and $y \oplus y^{\prime}$ in $M \oplus M^{\prime}$. 
The proof of (10): $B_{f, q} \otimes B_{f^{\prime} q^{\prime}}\left(\sum_{i} x_{i} \otimes x_{i}^{\prime}, \sum_{j} y_{j} \otimes y_{j}^{\prime}\right)=B_{f \otimes f^{\prime}, f^{2} \otimes q^{\prime}+q \otimes f^{\prime 2}+q \otimes q^{\prime}}$ $\left(\sum_{i} x_{i} \otimes x_{i}^{\prime}, \sum_{j} y_{j} \otimes y_{j}^{\prime}\right)=f \otimes f^{\prime}\left(\sum_{i} x_{i} \otimes x_{i}^{\prime}\right) f \otimes f^{\prime}\left(\sum_{j} y_{j} \otimes y_{j}^{\prime}\right)+B_{f 2 \bar{\otimes} q^{\prime}+q \bar{\otimes} f^{\prime 2}+q \otimes q^{\prime}}$ $\left(\sum_{i} x_{i} \otimes x_{i}^{\prime}, \sum_{j} y_{j} \otimes y_{j}^{\prime}\right)=\left(\sum f\left(x_{i}\right) f^{\prime}\left(x_{i}^{\prime}\right)\right)\left(\sum f\left(y_{j}\right) f^{\prime}\left(y_{j}^{\prime}\right)\right)+B_{f^{2} \otimes q^{\prime}}\left(\sum x_{i} \otimes x_{i}^{\prime}\right.$, $\left.\sum y_{j} \otimes y_{j}^{\prime}\right)+B_{q \bar{\otimes} f^{\prime 2}}\left(\sum x_{i} \otimes x_{i}^{\prime}, \quad \sum y_{j} \otimes y_{j}^{\prime}\right)+B_{q \otimes q^{\prime}}\left(\sum x_{i} \otimes x_{i}^{\prime}, \quad \sum y_{j} \otimes y_{j}^{\prime}\right)=$ $\sum_{i, j}\left(f\left(x_{i}\right) f\left(y_{j}\right) f^{\prime}\left(x_{i}^{\prime}\right) f^{\prime}\left(y_{j}^{\prime}\right)+f\left(x_{i}\right) f\left(y_{j}\right) B_{q^{\prime}}\left(x_{i}^{\prime}, y_{j}^{\prime}\right)+B_{q}\left(x_{i}, y_{j}\right) f^{\prime}\left(x_{i}^{\prime}\right) f^{\prime}\left(y_{j}^{\prime}\right)+\right.$ $\left.B_{q}\left(x_{i}, y_{j}\right) B_{q^{\prime}}\left(x_{i}^{\prime}, y_{j}^{\prime}\right)\right)=\sum_{i, j} B_{f, q}\left(x_{i}, y_{j}\right) B_{f^{\prime}, q^{\prime}}\left(x_{i}^{\prime}, y_{j}^{\prime}\right)$, for $\sum x_{i} \otimes x_{i}^{\prime}$ and $\sum y_{j} \otimes y_{j}^{\prime}$ in $M \otimes M^{\prime}$.

COROLlaRy 3. If $\langle M, f, q\rangle$ and $\left\langle M^{\prime}, f^{\prime}, q^{\prime}\right\rangle$ are non-degenerate extended quadratic modules, then $\langle M, f, q\rangle \perp\left\langle M^{\prime}, f^{\prime}, q^{\prime}\right\rangle$ is also non-degenerate. Furthermore, if $M$ and $M^{\prime}$ are finitely generated projective $A$ modules, then $\langle M, f, q\rangle \otimes\left\langle M^{\prime}, f^{\prime}, q^{\prime}\right\rangle$ is non-degenerate.

Remark 3. If 2 is inversible in the ring $A$, then the category Qua* $(A)$ is equivalent to the category Qua $(A)$, i.e. for any object $\langle M, f, q\rangle$ in $\operatorname{Qua}^{*}(A),\langle M, f, q\rangle \approx\left\langle M, 0, q+(1 / 2) f^{2}\right\rangle$.

Remark 4. Let $\left\langle M_{1}, f_{1}, q_{1}\right\rangle,\left\langle M_{2}, f_{2}, q_{2}\right\rangle$ and $\left\langle M_{3}, f_{3}, q_{3}\right\rangle$ be extended quadratic modules. Then we get the following natural isomorphisms in Qua* $(A)$;

1) $\left\langle M_{1}, f_{1}, q_{1}\right\rangle \perp\left\langle M_{2}, f_{2}, q_{2}\right\rangle \approx\left\langle M_{2}, f_{2}, q_{2}\right\rangle \perp\left\langle M_{1}, f_{1}, q_{1}\right\rangle$,

2) $\left\langle M_{1}, f_{1}, q_{1}\right\rangle \otimes\left\langle M_{2}, f_{2}, q_{2}\right\rangle \approx\left\langle M_{2}, f_{2}, q_{2}\right\rangle \otimes\left\langle M_{1}, f_{1}, q_{1}\right\rangle$,

3) $\left(\left\langle M_{1}, f_{1}, q_{1}\right\rangle \perp\left\langle M_{2}, f_{2}, q_{2}\right\rangle\right) \perp\left\langle M_{3}, f_{3}, q_{3}\right\rangle \approx\left\langle M_{1}, f_{1}, q_{1}\right\rangle \perp\left(\left\langle M_{2}, f_{2}, q_{2}\right\rangle\right.$ $\left.\perp\left\langle M_{3}, f_{3}, q_{3}\right\rangle\right)$,

4) $\left(\left\langle M_{1}, f_{1}, q_{1}\right\rangle \otimes\left\langle M_{2}, f_{2}, q_{2}\right\rangle\right) \otimes\left\langle M_{3}, f_{3}, q_{3}\right\rangle \approx\left\langle M_{1}, f_{1}, q_{1}\right\rangle \otimes\left(\left\langle M_{2}, f_{2}, q_{2}\right\rangle\right.$ $\left.\otimes\left\langle M_{3}, f_{3}, q_{3}\right\rangle\right)$,

5) $\left(\left\langle M_{1}, f_{1}, q_{1}\right\rangle \perp\left\langle M_{2}, f_{2}, q_{2}\right\rangle\right) \otimes\left\langle M_{3}, f_{3}, q_{3}\right\rangle \approx\left(\left\langle M_{1}, f_{1}, q_{1}\right\rangle \otimes\left\langle M_{3}, f_{3}, q_{3}\right\rangle\right)$ $\perp\left(\left\langle M_{2}, f_{2}, q_{2}\right\rangle \otimes\left\langle M_{3}, f_{3}, q_{3}\right\rangle\right)$,

6) $\left\langle M_{1}, f_{1}, q_{1}\right\rangle \otimes\langle A, I, 0\rangle \approx\left\langle M_{1}, f_{1}, q_{1}\right\rangle$.

Proof. We shall show only 5). For the other isomorphisms, we can see easily. To prove it, it is enough to show the identity

$\left(f_{1} \perp f_{2}\right)^{2} \bar{\otimes} q_{3}+\left(q_{1} \perp q_{2}-f_{1} \times f_{2}\right) \bar{\otimes} f_{3}^{2}+\left(q_{1} \perp q_{2}-f_{1} \times f_{2}\right) \bar{\otimes} q_{3}=$ $\left(f_{1}^{2} \bar{\otimes} q_{3}+q_{1} \bar{\otimes} f_{3}^{2}+q_{1} \otimes q_{3}\right) \perp\left(f_{2}^{2} \bar{\otimes} q_{3}+q_{2} \bar{\otimes} f_{3}^{2}+q_{2} \otimes q_{3}\right)-\left(f_{1} \otimes f_{3}\right) \times\left(f_{2} \otimes f_{3}\right)$. For any $\sum_{i}\left(x_{i}+y_{i}\right) \otimes z_{i}$ in $\left(M_{1} \oplus M_{2}\right) \otimes M_{3},\left(f_{1} \perp f_{2}\right)^{2} \otimes q_{3}+\left(q_{1} \perp q_{2}-f_{1} \times f_{2}\right)$ $\bar{\otimes} f_{3}^{2}+\left(q_{1} \perp q_{2}-f_{1} \times f_{2}\right) \otimes q_{3}\left(\sum_{i}\left(x_{i} \oplus y_{i}\right) \otimes z_{i}\right)=\sum_{i}\left[\left(f_{1}\left(x_{i}\right)+f_{2}\left(y_{i}\right)\right)^{2} q_{3}\left(z_{i}\right)+\right.$ $\left.\left(q_{1}\left(x_{i}\right)+q_{2}\left(y_{i}\right)-f_{1}\left(x_{i}\right) f_{2}\left(y_{i}\right)\right) f_{3}\left(z_{i}\right)^{2}+2\left(q_{1}\left(x_{i}\right)+q_{2}\left(y_{i}\right)-f_{1}\left(x_{i}\right) f_{2}\left(y_{i}\right)\right) q_{3}\left(z_{i}\right)\right]+$ $\sum_{i<j}\left[\left(f_{1}\left(x_{i}\right)+f_{2}\left(y_{i}\right)\right)\left(f_{1}\left(x_{j}\right)+f_{2}\left(y_{j}\right)\right) B_{q_{3}}\left(z_{i}, z_{j}\right)+\left(B_{q_{1}}\left(x_{i}, x_{j}\right)+B_{q_{2}}\left(y_{i}, y_{j}\right)-\right.\right.$ $\left.f_{1}\left(x_{i}\right) f_{2}\left(y_{j}\right)-f_{1}\left(x_{j}\right) f_{2}\left(y_{i}\right)\right) f_{3}\left(z_{i}\right) f_{3}\left(z_{j}\right)+\left(B_{q_{1}}\left(x_{i}, x_{j}\right)+B_{q_{2}}\left(y_{i}, y_{j}\right)-f_{1}\left(x_{i}\right) f_{2}\left(y_{j}\right)\right.$ 
$\left.\left.-f_{1}\left(x_{j}\right) f_{2}\left(y_{i}\right)\right) B_{q_{3}}\left(z_{i}, z_{j}\right)\right]=\sum_{i}\left[\left(f_{1}\left(x_{i}\right)^{2} q_{3}\left(z_{i}\right)+q_{1}\left(x_{i}\right) f_{3}\left(z_{i}\right)^{2}+2 q_{1}\left(x_{i}\right) q_{3}\left(z_{i}\right)\right)+\right.$ $\left.\left(f_{2}\left(y_{i}\right)^{2} q_{3}\left(z_{i}\right) q_{2}\left(y_{i}\right) f_{3}\left(z_{i}\right)^{2}+2 q_{2}\left(y_{i}\right) q_{3}\left(z_{i}\right)\right)-f_{1}\left(x_{i}\right) f_{3}\left(z_{i}\right) f_{2}\left(y_{i}\right) f_{3}\left(z_{i}\right)\right]+\sum_{i<j}\left[\left(f_{1}\left(x_{i}\right)\right.\right.$ $\left.f_{1}\left(x_{j}\right) B_{q_{3}}\left(z_{i}, z_{j}\right)+B_{q_{1}}\left(x_{i}, x_{j}\right) f_{3}\left(z_{i}\right) f_{3}\left(z_{j}\right)+B_{q_{1}}\left(x_{i}, x_{j}\right) B_{q_{3}}\left(z_{i}, z_{j}\right)\right)+\left(f_{2}\left(y_{i}\right) f_{2}\left(y_{j}\right)\right.$ $\left.B_{q_{3}}\left(z_{i}, z_{j}\right)+B_{q_{2}}\left(y_{i}, y_{j}\right) f_{3}\left(z_{i}\right) f_{3}\left(z_{j}\right)+B_{q_{2}}\left(y_{i}, y_{j}\right) B_{q_{3}}\left(z_{i}, z_{j}\right)\right)-\left(f_{1}\left(x_{i}\right) f_{3}\left(z_{i}\right) f_{2}\left(y_{j}\right)\right.$ $\left.\left.f_{3}\left(z_{j}\right)+f_{1}\left(x_{j}\right) f_{3}\left(z_{j}\right) f_{2}\left(y_{i}\right) f_{3}\left(z_{i}\right)\right)\right]=\left(f_{1}^{2} \bar{\otimes} q_{3}+q_{1} \otimes f_{3}^{2}+q_{1} \otimes q_{3}\right) \perp\left(f_{2}^{2} \bar{\otimes} q_{3}+\right.$ $\left.q_{2} \otimes f_{3}^{2}+q_{2} \otimes q_{3}\right)-\left(f_{1} \otimes f_{3}\right) \times\left(f_{2} \otimes f_{3}\right)\left(\sum_{i}\left(x_{i} \oplus y_{i}\right) \otimes z_{i}\right)$.

Definition. An extended quadratic module $\langle M, f, q\rangle$ is called hyperbolic if the associated bilinear module $\left(M, B_{f, q}\right)$ with $\langle M, f, q\rangle$ is hyperbolic, i.e. there exists an $A$-module $N$ such that $M=N \oplus N^{\prime}$ for some $A$-submodule $N^{\prime}, f(N)=q(N)=0$ and $N=N^{\perp}\left(=\left\{x \in M ; B_{f, q}(x, N)\right.\right.$ $=0\}$ ).

From Proposition 5 and the well known properties on bilinear modules, we get the following proposition.

Proposition 6.

1) If $\langle M, f, q\rangle$ and $\left\langle M^{\prime}, f^{\prime}, q^{\prime}\right\rangle$ are hyperbolic, then so is also $\langle M, f, q\rangle$ $\perp\left\langle M^{\prime}, f^{\prime}, q^{\prime}\right\rangle$.

2) If $M$ is a finitely generated projective $A$-module and $\langle M, f, q\rangle$ is hyperbolic then $\langle M, f, q\rangle$ is non-degenerate.

3) If $\langle M, f, q\rangle$ and $\left\langle M^{\prime}, f^{\prime}, q^{\prime}\right\rangle$ are non-degenerate and $\langle M, f, q\rangle$ is hyperbolic, then $\langle M, f, q\rangle \otimes\left\langle M^{\prime}, f^{\prime}, q^{\prime}\right\rangle$ is also hyperbolic.

\section{Extended Witt ring $W^{*}(A)$.}

From the argument in $\S 2$, we can construct a commutative ring $W^{*}(A)$. Let $\mathrm{Qua}_{p} *(A)$ be a full subcategory of Qua* $(A)$ consisting of non-degenerate extended quadratic modules with finitely generated projective modules. In the category $\mathrm{Qua}_{p} *(A)$, as well as the construction of the Witt ring $W(A)$, we consider the full subcategory $\mathrm{HQua}_{P}{ }^{*}(A)$ consisting of hyperbolic extended quadratic modules. And, using the notation of $K$-theory in [1], we define the extended Witt ring $W^{*}(A)$ by $W^{*}(A)=\operatorname{Coker}\left(K_{0}\left(\mathrm{HQua}_{p} *(A)\right) \rightarrow K_{0}\left(\right.\right.$ Qua $\left.\left._{p}{ }^{*}(A)\right)\right)$. Thus, it can be easily checked that $W^{*}(A)$ is a commutative ring with sum and product induced by orthogonal sum $\perp$ and tensor product $\otimes$. We denote by $[\langle P, f, q\rangle]$ the class of $\langle P, f, q\rangle$ in $W^{*}(A)$.

THEOREM 2. The extended Witt ring $W^{*}(A)$ has always the identity element $[\langle A, I, 0\rangle]$, and there exists a ring homomorphism of the Witt ring $W(A)$ to $W^{*}(A)$. Then, the image of $W(A)$ becomes an ideal of 
$W^{*}(A)$. If 2 is inversible in $A$, then it is an isomorphism; $W(A) \underset{\approx}{\longrightarrow}$ $W^{*}(A)$.

Proof. Let $\mathrm{Qua}_{p}(A)$ be the full subcategory of Qua $(A)$ consisting of non-degenerate quadratic modules $(P, q)$ with finitely generated projective $A$-module $P$, and $\operatorname{HQua}_{p}(A)$ the full subcategory of $\mathrm{Qua}_{p}(A)$ whose objects are hyperbolic in $\operatorname{Qua}_{p}(A)$. Consider the functor $\Phi:$ Qua $_{p}(A) \rightarrow$ Qua $_{p} *(A) ;(P, q) \sim\langle P, 0, q\rangle$, then we have the following commutative diagram

$$
\begin{aligned}
& K_{0}\left(\operatorname{HQua}_{p} *(A)\right) \rightarrow K_{0}\left(\operatorname{Qua}_{p} *(A)\right) \rightarrow W^{*}(A) \rightarrow 0 \\
& \uparrow K_{0}(\Phi) \quad \uparrow K_{0}(\Phi) \\
& K_{0}\left(\operatorname{HQua}_{p}(A)\right) \rightarrow K_{0}\left(\operatorname{Qua}_{p}(A)\right) \rightarrow W(A) \rightarrow 0
\end{aligned}
$$

where two rows are exact.

Thus, the ring homomorphism $K_{0}(\Phi)$ induces a ring homomorphism $\omega: W(A) \rightarrow W^{*}(A)$. Then, Im $\omega$ becomes an ideal of $W^{*}(A)$, for $[\langle P, f, q\rangle]$ $\left[\left\langle P^{\prime}, 0, q^{\prime}\right\rangle\right]=\left[\left\langle P \otimes P^{\prime}, 0, q \otimes q^{\prime}+f^{2} \otimes q^{\prime}\right\rangle\right]$ in $W^{*}(A)$. If 2 is inversible in $A$, by Remark $3, K_{0}(\Phi)$ is an isomorphism, therefore, so is also $\omega: W(A) \underset{\sim}{\longrightarrow} W^{*}(A)$.

\section{The unit group of $W^{*}(A)$ and $Q_{s}(A)$.}

In this section, we consider a relation between the separable quadratic extension group $Q_{s}(A)$ and the unit group $U\left(W^{*}(A)\right.$ ) of the extended Witt ring $W^{*}(A)$.

THEOREM 3. There exists a group homomorphism of $Q_{s}(A)$ to $U\left(W^{*}(A)\right)$;

$$
\Theta: Q_{s}(A) \longrightarrow U\left(W^{*}(A)\right) ;[U, f, q] \sim[\langle U, f, 2 q\rangle] .
$$

Proof. Let $[U, f, q]$ be an element in $Q_{s}(A)$. By Theorem 1 , the bilinear module $\left(U, D_{f, q}\right)$, called the discriminant of $[U, f, q]$, is nondegenerate. Since $D_{f, q}(x, y)=f(x) f(y)+2 B_{q}(x, y)=f(x) f(y)+B_{2 q}(x, y)$ $=B_{f, 2 q}(x, y)$ for any $x$ and $y$ in $U$, we have $D_{f, q}=B_{f, 2 q}$. Therefore, $\langle U, f, 2 q\rangle$ is in $\mathrm{Qua}_{p}{ }^{*}(A)$. Now, we shall show that $\Theta$ is well defined: If $[U, f, q]=\left[U^{\prime}, f^{\prime}, q^{\prime}\right]$ is in $Q_{s}(A)$, then there exist an $A$-isomorphism $\sigma: U \rightarrow U^{\prime}$ and an $A$-homomorphism $g: U \rightarrow A$ such that $q^{\prime} \circ \sigma=q+f g-g^{2}$ and $f^{\prime} \circ \sigma=f-2 g$. Then, we get $2 q^{\prime} \circ \sigma=2 q+2 f g-2 g^{2}$ and $f^{\prime} \circ \sigma=$ 
$f-2 g$, that is, $\langle U, f, 2 q\rangle \approx\left\langle U^{\prime}, f^{\prime}, 2 q^{\prime}\right\rangle$ in Qua $_{p} *(A)$. Thus, the map $\Theta: Q_{s}(A) \rightarrow W^{*}(A) ;[U, f, q] \sim[\langle U, f, 2 q\rangle]$ is well defined. Furthermore, we have

$$
\Theta\left([U, f, q]\left[U^{\prime}, f^{\prime}, q^{\prime}\right]\right)=\Theta\left(\left[U \otimes U^{\prime}, f \otimes f^{\prime}, f^{2} \bar{\otimes} q^{\prime}+q \bar{\otimes} f^{\prime 2}+2 q \otimes q^{\prime}\right]\right)=
$$
$\left[\left\langle U \otimes U^{\prime}, f \otimes f^{\prime}, f^{2} \bar{\otimes} 2 q^{\prime}+2 q \bar{\otimes} f^{\prime 2}+2 q \otimes 2 q^{\prime}\right\rangle\right]=[\langle U, f, 2 q\rangle] \cdot\left[\left\langle U^{\prime}, f^{\prime}, 2 q^{\prime}\right\rangle\right]$, and $\Theta([A, I, 0])=[\langle A, I, 0\rangle]$. Accordingly, $\operatorname{Im} \Theta$ is contained in $U\left(W^{*}(A)\right)$ and $\Theta: Q_{s}(A) \rightarrow U\left(W^{*}(A)\right)$ is a group homomorphism.

Remark 5.

1) if $K$ is a field with the characteristic $\neq 2$, then $U\left(W^{*}(A)\right)=$ $U(W(A)) \approx U(K) / U(K)^{2}, Q_{s}(K) \approx U(K) / U(K)^{2}$ and $\Theta$ is an isomorphism.

2) If $K$ is a field with characteristic 2 , then $\Theta$ is a zero homomorphism.

\section{REFERENCES}

[1] H. Bass: Lecture on Topics in Algebraic K-theory, Tata Institute of Fundamental Research, Bombay, 1967.

[2] P. M. Cohn: Quadratic extensions of skew fields, Proc. London Math. Soc. 11 (1961) 531-556.

[3] T. Kanzaki: On commutor ring and Galois theory of separable algebras, Osaka J. Math. 1 (1964).

[4] T. Kanzaki: Generalized crossed product and Brauer group, Osaka J. Math. 5 (1968) 175-188.

[ 5 ] T. Kanzaki: On bilinear module and Witt ring over a commutative ring, Osaka J. Math. 8 (1971) 485-496.

[6] P. A. Micali and O. E. Villamayor: Algebra de Clifford et groupe de Brauer, Ann. scient. Ec. Norm. Sup., t. 4 (1971) 285-310.

[ 7 ] P. P. Revoy: Sur les deux premiers invariants d'une forme quadratique, Ann. scient. Ec. Norm. Sup., t. 4 (1971) 311-319.

Osaka City University 